\title{
Interoperability Performance Assessment of Multivendor IEC61850 Process Bus
}

DOI:

10.1109/TPWRD.2015.2509644

\section{Document Version}

Accepted author manuscript

Link to publication record in Manchester Research Explorer

\section{Citation for published version (APA):}

Crossley, P., Chen, X., \& Guo, H. (2016). Interoperability Performance Assessment of Multivendor IEC61850

Process Bus. IEEE Transactions on Power Delivery, 31(4), 1934-1944. [1].

https://doi.org/10.1109/TPWRD.2015.2509644

\section{Published in:}

IEEE Transactions on Power Delivery

\section{Citing this paper}

Please note that where the full-text provided on Manchester Research Explorer is the Author Accepted Manuscript or Proof version this may differ from the final Published version. If citing, it is advised that you check and use the publisher's definitive version.

\section{General rights}

Copyright and moral rights for the publications made accessible in the Research Explorer are retained by the authors and/or other copyright owners and it is a condition of accessing publications that users recognise and abide by the legal requirements associated with these rights.

\section{Takedown policy}

If you believe that this document breaches copyright please refer to the University of Manchester's Takedown Procedures [http://man.ac.uk/04Y6Bo] or contact uml.scholarlycommunications@manchester.ac.uk providing relevant details, so we can investigate your claim.

\section{OPEN ACCESS}




\title{
Interoperability Performance Assessment of Multivendor IEC61850 Process Bus
}

\author{
Xi Chen, Student Member, IEEE, Hao Guo, Student Member, IEEE and Peter Crossley, Senior Member, IEEE
}

\begin{abstract}
Substations are increasingly reliant on devices compatible with IEC61850 standards. However, the conformity of a device with the standards does not necessarily guarantee interoperability with devices from different manufacturers. The use of devices compatible with IEC61850 standards presents a challenge to many system integrators, especially due to lack of familiarity with features such as Generic Object Oriented Substation Event (GOOSE), reporting, sample values and the need for system redundancy. To facilitate a smooth implementation, all the features and the data exchanges between devices need to be tested to ensure the system operates correctly. It is important to determine the compatibility of the devices made by different manufacturers.
\end{abstract}

This paper presents a technique to assess the performance of Merging Units from different manufacturers, when operating with Intelligent Electronic Devices (IEDs) performing a distance protection function. The performance of the process bus with parallel redundancy protocol is evaluated using a closed loop approach involving a Real Time Digital Simulator.

Index Terms-- IEC61850, Interoperability, Parallel Redundancy, Performance Evaluation, Substation Automation System.

\section{INTRODUCTION}

$\mathrm{N}$ UMEROUS protection and control systems for transmission substations are reaching the end of their asset life [1]. This provides an opportunity to adopt new technologies and architectures capable of delivering economically appropriate solutions with a long operating life. A globally appropriate solution involves the direct implementation of IEC61850 standards and includes Ethernet based process bus architectures for high-speed peer-to-peer communications between the primary plant and the secondary systems. The secondary systems will be entirely digitized and all existing copper "signal" connections replaced with fibre optic links. Merging Units (MUs), located at the secondary terminals of the primary plant, communicate via the process bus to IEDs using Sampled Values (SVs) [2] [3] and GOOSE messages [4]. The architecture facilitates interoperability between IEDs from different vendors, if they conform to the IEC61850 standards [5]. The overall goal is to deliver significant benefits to the operation, expandability and lifetime cost of future substations by delivering a plug and play

Xi Chen is Ph.D. with EEPS research group, Department of Electrical Engineering, Ferranti Building, Manchester University, Manchester, M1 3BB, UK (e-mail: xi.chen-11@ postgrad.manchester.ac.uk).

Hao Guo is Ph.D. with EEPS research group, Department of Electrical Engineering, Ferranti Building, Manchester University, Manchester, M1 3BB, UK (e-mail: hao.guo-5@postgrad.manchester.ac.uk).

Peter Crossley is Professor of Power Systems, Director of Doctoria Training Centre, Ferranti Building, Manchester University, Manchester, M1 3BB, UK (email: p.crossley@ manchester.ac.uk) approach to secondary systems [6]. Manufacturers usually conduct interoperability testing within their own product lines; consequently, coordination and data privacy are usually not an issue. However, interoperability between devices and systems from multiple vendors are usually not fully evaluated [7].

One often quoted concern is that MUs and IEDs designed by different vendors may not identically interpret the standards and this might cause problems in the future. Performance evaluation reported in many previous papers was based on simulation tools, such as Optimized Network Engineering Tools (OPNET) [8], and the interoperability performance of commercial devices is largely unknown. Consequently, many utilities are not confident in the full adoption of the standards, and especial concern is given to the process bus. Numerous validation exercises have been conducted to assess the performance of SV and GOOSE on systems supplied by a single vendor [9]-[12], or a small group of collaborating vendors [13]-[15]; but limited interoperability results have been presented for complex systems that involve multiple devices from different vendors. An experimental approach is proposed in this paper, which assesses and analyses the interoperability performance of an IEC 61850 process bus architecture utilising devices supplied by four major vendors. The simulated system used in this study, as shown in Fig. 1, involved modelling in a Real Time Digital Simulator (RTDS) the effect on a $400 \mathrm{kV}$ mesh substation A of a series of short circuit faults on the feeder to substation B.

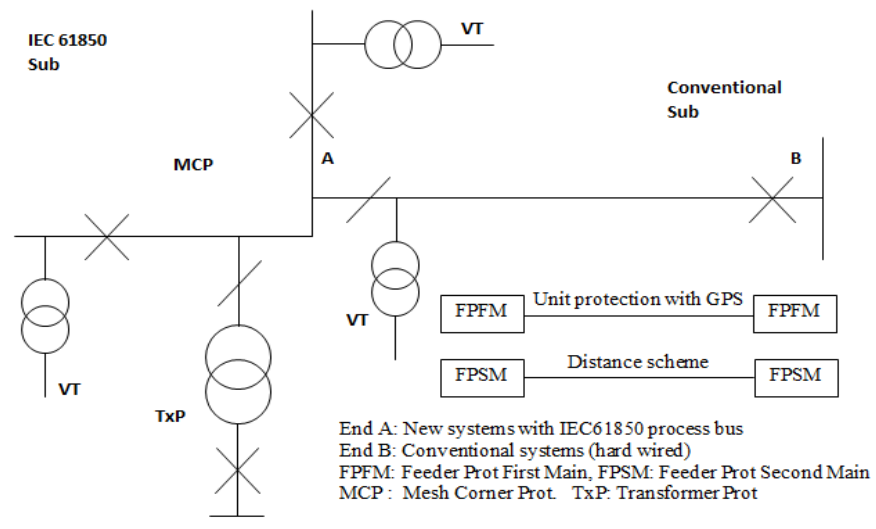

Fig. 1. Test 400kV Substation Architecture Diagram modelled in RTDS.

The objective was to understand how the performance of IEDs and MUs supplied by different vendors, and operating in the simulated substation A, performed when configured within a feeder protection scheme with conventional relays at B. A single bay process bus, with Ethernet Switches, was built in the laboratory and used to connect MUs and IEDs from different vendors. The SV, GOOSE messages and the 
IEEE1588 Precision Time Protocol (PTP) [16] data were communicated over the same process bus and various evaluation tests were performed. This included the normal IED operational test, the MU performance test, the impact of network traffic and SV loss on IEDs, and an operating performance assessment on the network architecture with Parallel Redundancy Protocol (PRP) [17] protocol.

\section{TEST MethoD}

The IEDs used for this study are configured as distance protection relays, one with SV (9-2 LE) inputs from manufacturer A, one with SV inputs from manufacturer $\mathrm{C}$ and the other with conventional analogue current transformer and voltage transformer inputs $(110 \mathrm{~V}$ and $1 \mathrm{~A})$ from manufacturer A. This allowed a performance comparison between conventional and SV inputs to be performed. The test system consists of a RTDS simulator, OMICRON amplifiers, a traffic generator/impairment device, an Ethernet tap and various panels of IEDs, MUs and Ethernet switches from different vendors. The paper refers to these as A-IED, A-MU, B-MU, $\mathrm{C}$-IED and C-MU. The test bed enables the performance of Ethernet switches, MUs and IEDs to be assessed whilst introducing network traffic and impairment.

The RTDS is equipped with the GTNET (Gigabit Transceiver Network) card, configured with GOOSE capability (referred to as GTNET-GSE). The RTDS provides closed loop testing by generating the voltage and current signals, via the amplifiers, to the MUs. The signals are then digitised and sent as SVs to the IEDs, which are processed and when appropriate converted into a GOOSE "trip" message, subscribed to by the RTDS. The operating performance of a 92LE process bus, with devices from different vendors, is detailed in the next section.

The protection settings were consistent with those protecting a transmission line in a real substation. The Mho curve is shown graphically in Fig. 2. The three zone boundary is configured as $80 \%, 150 \%$ and $250 \%$ for Zone 1, Zone 2 and Zone 3 respectively. The protection scheme logic delays are $0 \mathrm{~s}$ for Zone 1, 500ms for Zone 2 and 1000ms for Zone 3.

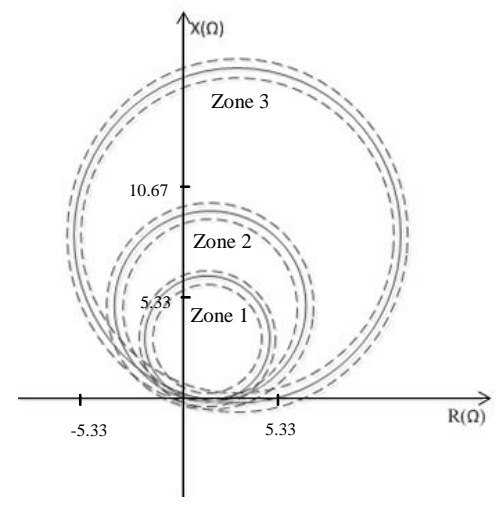

Fig. 2. IED distance protection three zone setting diagram.

The performance of the multivendor system was assessed by simulating faults at different locations along the transmission feeder and measuring the elapsed time from fault occurrence to the receipt of a differential protection operation indication via a GOOSE message or contact input to the RTDS simulator.

\section{A. Measurement validation}

The tests with conventional relays were first carried out to provide a benchmark for the measurement system. The variation in response time of the conventional relay from manufacturer $\mathrm{A}$ was assessed by injecting the original secondary voltage and current directly to the relay in normal system operating conditions and then during fault conditions. The results were used to compare the performance of SV and GOOSE signals in the multi-vendor system.

\section{B. Merging Unit Processing and Publishing Delay}

The Merging Unit acquires analogue values and converts them to digital values. The converting process time for different MUs is measured between the fault occurrence time and the time when the first SV packet containing the fault current value is generated. The fault occurrence is controlled by the 1 pulse per second (1-PPS) time signal. The RTDS simulator is equipped with the GTSYNC card and the time signal is extracted from this card which is synchronised with the 1-PPS signal from the external clock source. The pulse signal is programmed to trigger the fault occurrence. The time source of the 1-PPS signal for synchronising the RTDS is used to synchronise the MU. The time source can provide different time synchronisation signals, such as IEEE 1588, IRIG-B (Inter-Range Instrumentation Group - Time Code Format B) and 1-PPS signal and the MUs can be configured to be synchronised by different types of time signals. Each IEC61850-9-2 SV message includes a sample counter, namely, SmpCnt and the synchronising of SV is realised by resetting the SmpCnt parameter value to 0 when the time synchronising signal occurs. The SmpCnt value will range from 0 to 3999 in $50 \mathrm{~Hz}$ power system for protection applications using 9-2LE with 80 samples per nominal cycle. The inter frame time between each SV packet is $250 \mu$ s at $50 \mathrm{~Hz}$. The fault occurred at the same time when the SmpCnt is reset to 0 in the SV packet. The waveform of the captured SV was recovered point by point using Message Analysis Software [18].

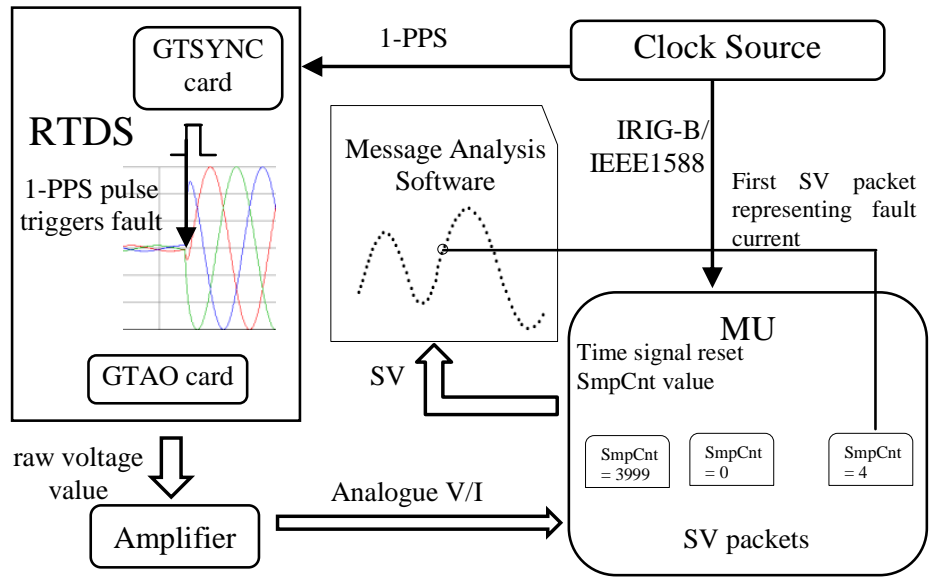

Fig. 3. Merging Unit processing delay measurement test setup.

The position on the waveform is mapped to the individual captured packets. The first packet containing the fault current value is extracted and the SmpCnt value of that packet, e.g. 4, 
is recorded. The processing time is equal to the time period between the SmpCnt $=0$ packet and the first fault current SV packet. The test setup and operating concept is shown in Fig. 3 below.

The method to measure the Merging Unit publication time is through the use of the Ethernet capture card with externally synchronised time stamping. The Endace DAG7.5G4 card is a four-port card that can time-stamp the received frames. The DAG card is capable of capturing or transmitting four 1000 $\mathrm{Mb} / \mathrm{s}$ Ethernet streams, and includes a facility to synchronise its time-stamping clock to an external 1-PPS source [19]. The time-stamping clock is integral to the Ethernet capture hardware, giving an absolute error of $\pm 100 \mathrm{~ns}$ from the 1-PPS reference and a relative error of \pm 8 ns between the four capture ports [20]. The card recorded the time when an Ethernet frame is received and a time stamp is then tagged to the captured frame. The Merging unit and capture card are synchronised to the same time source. The SV packet whose SmpCnt $=0$ is taken to measure the time used for the SV processing delay as it is the only sample precisely aligned to the synchronisation source [9]. Previous work [21] has clearly illustrated how the SV from the RTDS publication delay is measured. This range of tests could be extended to include MUs from different manufacturers. The propagation delay of the signal through Ethernet cable is 5-6ns per meter which can be ignored since the cable distance is very short [22]. The Test setup is shown in Fig. 4.

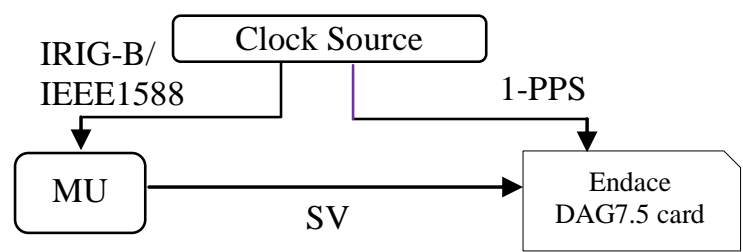

Fig. 4. Merging Unit publication delay measurement test setup.

The SV packet with SmpCnt $=0$ appears when 1-PPS signal arrives. Over a ten minute period, more than 600 measurements were taken for calculation and the timestamp recorded. The captured frames were filtered with the criterion SmpCnt $=0$. The "appearance delay" was then determined by taking the fractional second component of each frame's timestamp. This gives the total time taken from the occurrence of the 1-PPS synchronising signal to the appearance of the frame on the Ethernet [20].

\section{Interoperability Test without Background Traffic}

To verify the interoperability, distance protection functionality with different architecture of MUs and IEDs is used. Three MUs and two IEDs give six test cases for comparison purposes as shown in Table I. RTDS subscribes to the GOOSE trip messages through the GTNET-GSE card. The MUs and IEDs are connected directly without any Ethernet switches in between. The RTDS RunTime plot recorded the time between the fault occurrence and the reception of GOOSE signals. The single line diagram of operation of Zone 1 is shown in the Appendix.

TABLE I

List of MUs versus IEDs

\begin{tabular}{|c|c|}
\hline MU type & Subscriber IEDs \\
\hline A-MU & A-IED \\
\hline
\end{tabular}

\begin{tabular}{|c|c|}
\hline & C-IED \\
\hline \multirow{2}{*}{ B-MU } & A-IED \\
\cline { 2 - 2 } & C-IED \\
\hline \multirow{2}{*}{ C-MU } & A-IED \\
\cline { 2 - 2 } & C-IED \\
\hline
\end{tabular}

D. Network Architecture Test with Parallel Redundancy Protocol (PRP)

Communication architecture has a profound influence on the performance of the substation automation system, as well on the interoperability of the devices connected to the network. A simple PRP network is setup with Ethernet switches as shown in Fig. 5 below. The Red Box is equipped with parallel redundancy protocol. The input signal to the Red Box SV from MU is injected from the Red Box 1. The input SV packet is duplicated and two identical copies is transmitted through $\mathrm{LAN}_{1}$ (Local Area Network) and $\mathrm{LAN}_{2}$ simultaneously. The first packet arriving at Red Box 2 is maintained and forwarded whilst the second packet is discarded. The traffic is injected at different locations, either $\mathrm{LAN}_{1}$ or $\mathrm{LAN}_{2}$ to see the influence on the SV transmission. The SV is injected from the Red Box 1 and with $80 \mathrm{Mb} / \mathrm{s}$, $100 \mathrm{Mb} / \mathrm{s}, 120 \mathrm{Mb} / \mathrm{s}$ and $200 \mathrm{Mb} / \mathrm{s}$ background traffic. Note: the bandwidth of each switch is $100 \mathrm{Mb} / \mathrm{s}$.

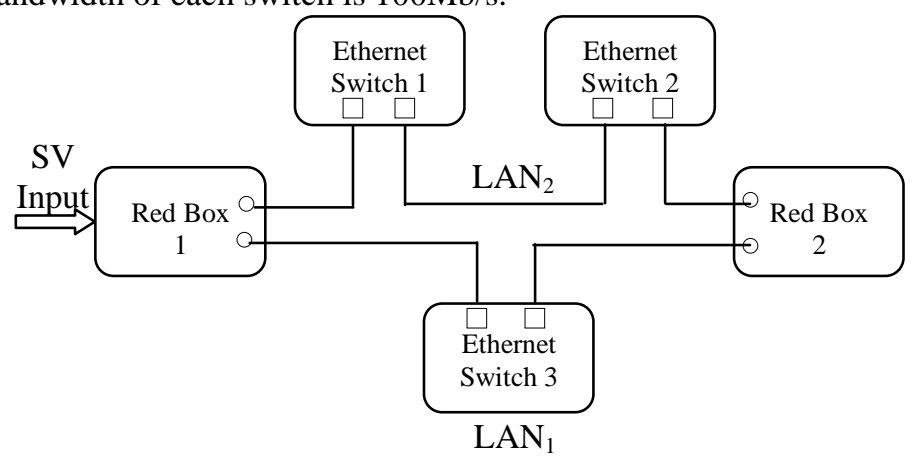

Fig. 5. Schematic diagram of PRP network with Ethernet Switches and Redundancy Boxes.

\section{1) : Unicast Background Traffic}

The test setup is shown in Fig. 6 and the network architecture is shown in Fig. 5. Unicast Traffic was created in the traffic generator and connected to the Ethernet switches at $\mathrm{LAN}_{2}$. The traffic generator has 4-ports available for generating the traffic [23]. The unicast traffic with destination address configured to port 2 is injected from port 1 to the network using Ethernet switch 1. The traffic goes into the network from Ethernet switch 1 and flows back to the traffic generator port 2 which is connected to Ethernet switch 2. The traffic in $\mathrm{LAN}_{2}$ does not go through the Red Box 2 and thus does not influence $\mathrm{LAN}_{1}$. Even if $\mathrm{LAN}_{2}$ is overloaded, SV does not lose any packets. However, if the unicast traffic destination address is not within the network, unicast traffic will flood the whole network in the same way as the multicast traffic. The Ethernet Tap copied the input SV and output two identical streams; one is directly connected to the Ethernet capture card and the other goes through the network before being captured by the Ethernet capture card. The time difference between the same packets captured by the two ports is the time delay in the network. The Ethernet capture card is connected to the Red Box 2 of the PRP network. 


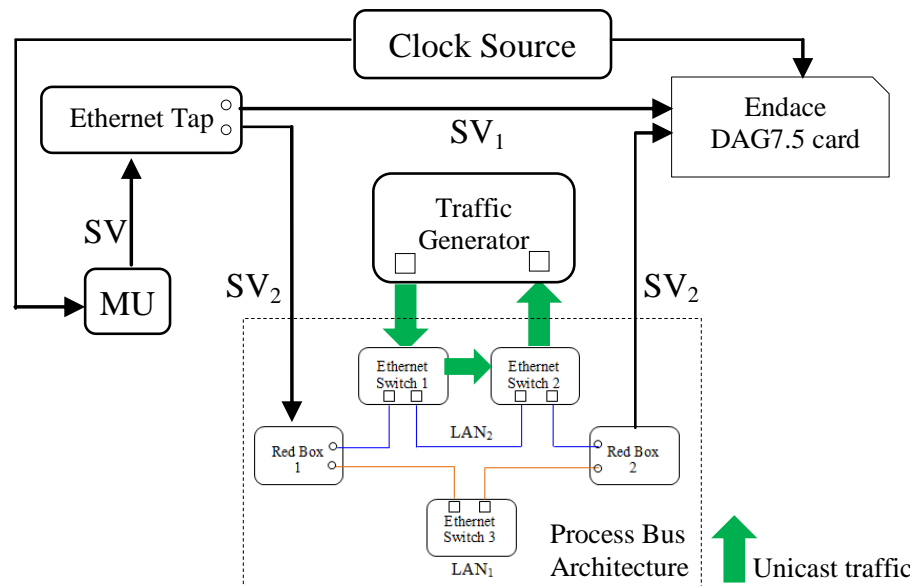

Fig. 6. Test set up for messages delay measurement with background traffic in PRP network.

With the PRP protocol in both of the Red Boxes, the SV has two routes in the network, $\mathrm{LAN}_{1}$ and $\mathrm{LAN}_{2}$. The tests used to compare the transmission time of $\mathrm{SV}$ in the network were carried out with $\mathrm{LAN}_{1}$ connected to Red Box and without $\mathrm{LAN}_{1}$.

\section{2) : Multicast Background Traffic}

Multicast traffic is injected into the Ethernet switches in both $\mathrm{LAN}_{1}$ and $\mathrm{LAN}_{2}$. Unlike unicast traffic, multicast traffic travels around the whole network. The SV delay is investigated when the traffic injects at different positions on the network.

\section{E. Sampled Value Loss Impact on IEDs}

Ethernet networks sometimes lose frames due to network contingency, but an IED is designed to tolerate the loss of "some" SV packets to ensure protection performance is maintained [24]. However, there is a limit on the loss rate value, i.e. where the IED cannot read the SV correctly. The test is carried out to assess the ability of IEDs from different manufacturers to cope with SV packet loss. The Traffic Impairment Emulator is used to simulate the frame loss situation to discard the packets of SV stream, as shown in Fig V 7. Different loss rates of the SV stream can be set by selecting the number of frames to be discarded within a period.

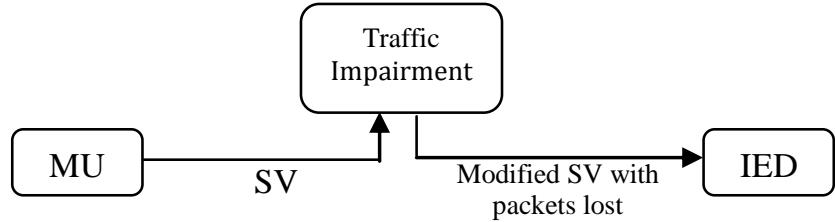

Fig. 7. SV loss impact evaluation on IED test system setup.

\section{F. Frequency Tracking Capability of MUs}

The MU filter performance test is used to investigate the bandwidth of the MU or the "waveshape quality" of the original analogue signals that the MU is able to recover. A "light edition" Merging Unit samples the signal at $4 \mathrm{k}$ samples/second, hence ideally it should be able to recover frequencies in the original signal up to $2 \mathrm{kHz}$. A sinusoidal voltage signal was injected into both MUs at frequencies from $10 \mathrm{~Hz}$ to $2000 \mathrm{~Hz}$ and the voltage ratio between the SV and the original signal recorded and plotted. The tests indicated A-MU and $\mathrm{B}-\mathrm{MU}$ have different performances compared to $\mathrm{C}-\mathrm{MU}$ when capturing signals at higher frequencies.

\section{$G$. The Recovery of the Step Change Signal}

The step change performance of each MU is assessed by applying a $25 \mathrm{~V}$ step change voltage to the analogue input of the MU. The output SV packets were captured via Wireshark and the waveform restored by the Message Analysis Software. Fig. 8 shows the actual step change analogue input to the MUs.

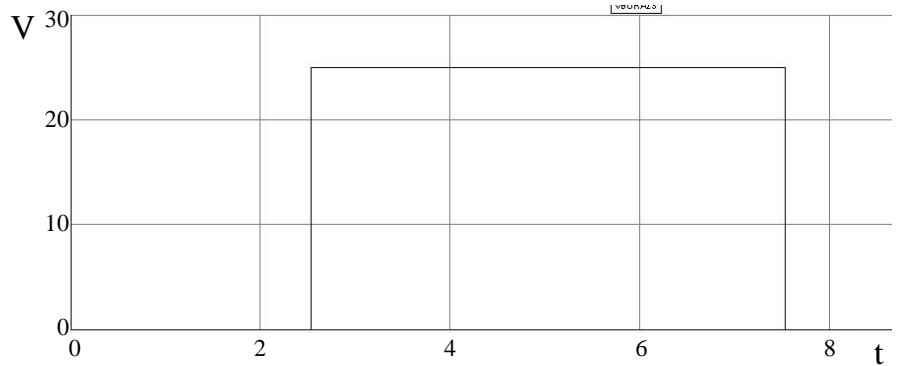

Fig. 8. 25V step change input signal shown on RTDS runtime.

\section{H. Network Background Traffic Impact on IEDs}

Ethernet switch 1 and Ethernet switch 2 in Fig. 5 are connected to traffic generator port 1 and port 2. The Merging Unit and A-IED are connected to the Red Box 1 and Red Box 2 respectively in the Fig. 5 process bus architecture. The SV packets were captured and recovered point-by-point in the Message Analysis Software. Fig. 9 is a screenshot of waveform recovery for a short period of time. The period of sinewave is $20 \mathrm{~ms}$. All the points were lined up to make the continuous sinewave. Fig. 9 (a) is the SV output from the traffic impairment, where the SV packets are discarded equally according to the setting, e.g. for a $5 \%$ loss rate setting, 5 consecutive packets are discarded every 100 packets. However, when the background traffic exceeds the bandwidth of the Ethernet switches, some of the packets are delayed and more are lost. As shown in Fig. 9 (b), point a should be located at the position of point $\mathbf{b}$ on the sinewave. Thus there are some sparks along the waveform where the packets are delayed and the points should be placed on the sinewave.

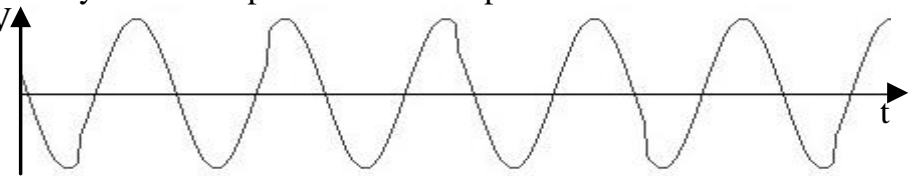

(a) SV waveform with $6 \%$ loss by traffic impairment.

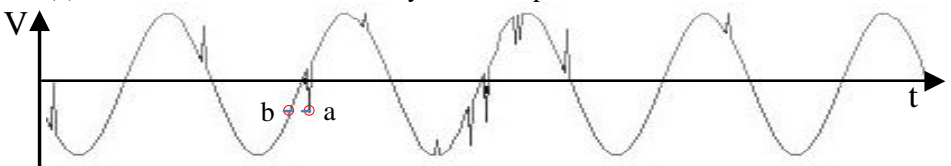

(b) SV waveform with $6 \%$ loss by background traffic.

Fig. 9. Comparison between distorted SV streams due to traffic impairment and background traffic.

The test setup is as shown in Fig. 9. All the background traffic goes through Red Box 2 and the SV packets are discarded when the Red Box 2 buffer is full. If the background traffic priority is less than SV, the SV does not lose packets. If the priority of background traffic is equal or higher than SV, the SV gets lost. In this test, the background traffic has the same priority as the SV. The SV input as shown in Fig. 10 left does not transmit successfully and the output 
looks like SV output waveform on the right. The influence of SV loss caused by background traffic on the IED is detailed in section III part $\mathrm{H}$.

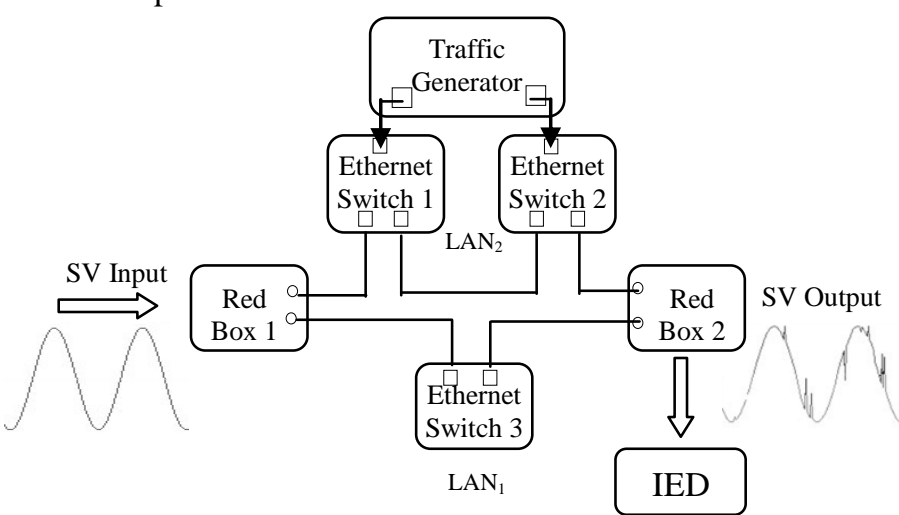

Fig. 10. SV loss impact on IED evaluation test system setup.

\section{Overwrite Impact on IEDs}

Overwriting of the SV streams is tested by overwriting specific portions of the data as shown in Fig. 11. The blue part is the overwritten part in each packet.

SV packets

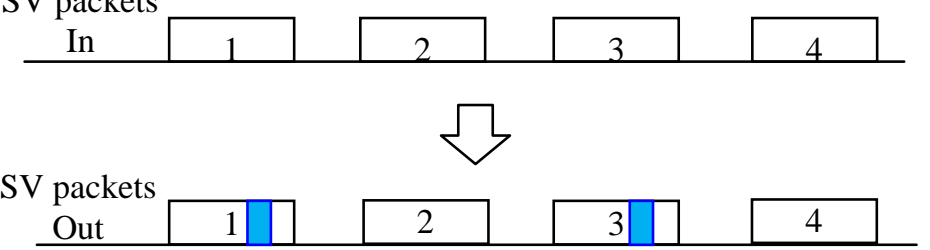

Fig. 11. Overwrite of SV packets conceptual diagram.

The SV is multicast with the destination address 01:0c:cd:04:00:01. When the SV goes through traffic impairment, a certain number of the SV packets' destination MAC address is overwritten to 01:0d:cd:03:00:01. Fig. 11 shows $50 \%$ of the packets are overwritten. The impaired SV streams are transmitted to A-IED and C-IED and the response of the two IEDs differs from each other.

\section{J. Time Synchronisation Loss \& Regain Impact on MUs}

The behaviour of the MU when losing and regaining the time synchronisation signal was tested by looking at the sample count attribute in the SV packet. The results are detailed in Section III part J.

\section{Test RESUlts}

\section{A. Measurement validation}

Three-phase to ground faults were used to test the operation of the relay. The faults were located at $40 \%, 120 \%$ and $180 \%$ on the transmission line, triggering Zone1, Zone 2 and Zone3 protection scheme individually. The binary trip signal was wired from relay contact output to RTDS GTFPI (GigabitTransceiver Front Panel Interface) card. The GTFPI card connects to one low voltage digital input interface panel [25]. It can work as the RTDS interface for binary input signals. The binary trip signal reception time was assessed by applying 100 faults to the relay. The average time and the standard deviation of the 100 tests were recorded on the RTDS RunTime plot, as shown in TABLE II.
TABLE II

Conventional Distance Relay Response Time (ms)

\begin{tabular}{|c|c|c|c|}
\hline & Zone1 & Zone2 & Zone3 \\
\hline Average & 47.317 & 557.45 & 1019.933 \\
\hline $\begin{array}{c}\text { Standard } \\
\text { Deviation }\end{array}$ & 6.362 & 7.713 & 3.558 \\
\hline Maximum & 54.379 & 574.164 & 1037.652 \\
\hline
\end{tabular}

\section{B. Merging Unit Processing and Publishing Delay}

The Merging Unit processing delay tests were carried out 100 times with each MU synchronised to different types of time signals. The results are shown in TABLE III and Fig. 12.

TABLE III

SV Processing Time of Multivendor MUs with different time synchronisation methods

\begin{tabular}{|c|c|c|c|c|}
\hline \multirow{2}{*}{} & \multicolumn{3}{|c|}{ SmpCnt } & Processing Time $(\mu \mathrm{s})$ \\
\cline { 2 - 5 } & Average & Maximum & Average & Maximum \\
\hline A-MU with IEEE1588 & 3.07 & 5 & 767.5 & 1250 \\
\hline A-MU with IRIG-B & 2.86 & 4 & 720 & 1000 \\
\hline B-MU with GPS & 4.28 & 7 & 1067.5 & 1750 \\
\hline C-MU with IEEE1588 & 3.64 & 6 & 910 & 1500 \\
\hline C-MU with IRIG-B & 3.29 & 5 & 827.5 & 1250 \\
\hline
\end{tabular}

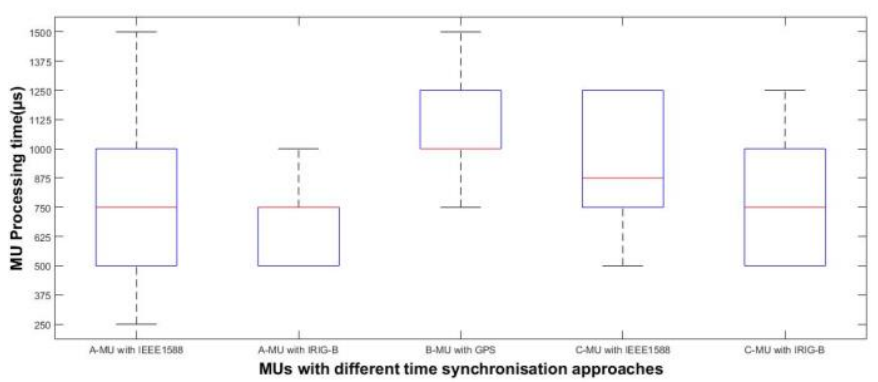

Fig. 12. SV Processing Time of Multivendor MUs with different time synchronisation methods

The average SmpCnt value in the first fault current packet from A-MU is 3.07 when synchronised with IEEE1588 and 2.86 when synchronised with IRIG-B. For C-MU, the average result is 3.64 and 3.29 when synchronised to the above two time signals. B-MU has only one option for time synchronisation which is GPS signal and the average value for SmpCnt is 4.28 . At 80 sampled per $50 \mathrm{~Hz}$ cycle (packets $250 \mu \mathrm{s}$ apart), the average processing delay for A-MU is $0.72-0.77 \mathrm{~ms}$, while C-MU needs $0.83-0.91 \mathrm{~ms}$ to process the analogue signal. B-MU takes $1.068 \mathrm{~ms}$ to process the signals. A-MU has the shortest processing time among the three MUs. For the maximum processing delay, A-MU and C-MU only have less than a two packet difference between 4 and 6, which is $1000 \mathrm{~ns}$ to $1500 \mathrm{~ns}$ respectively. $\mathrm{B}-\mathrm{MU}$ has the longest time which is 1750 ns.

The time difference between the 1-PPS pulse and the arrival of the SV frame with SmpCnt $=0$ is recorded in Fig. 13 below. The packet size of different MUs is different. A-MU publishes a 133 byte packet and B-MU and C-MU 129 byte and 134 byte packets respectively.

For each MU, the publication time is almost the same, no matter which signal is used for time synchronisation. When comparing the three MUs, B-MU has the longest publication time. As it is normally used for distribution network applications, B-MU has a separate interface for capturing voltage and current signals from VT/CT. The interface transmits the raw data to the merging unit which makes the SV 
packets and these are then published to the network. A-MU is about $84 \mu$ s quicker than the $\mathrm{C}-\mathrm{MU}$ in the publication process.

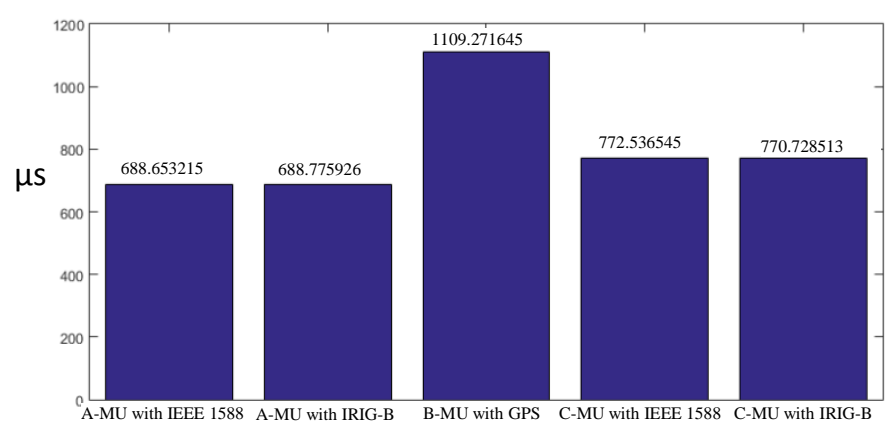

Fig. 13. SV Publication Time of Multivendor MUs with different time synchronisation methods.

\section{Interoperability Test without Background Traffic}

Tests were carried out 100 times to calculate the average and standard deviation values for the selected three-phase balanced fault in each of the three zones. Conventional test results from Table II are included in the Table IV below for comparison.

TABLE IV

Multi-Vendor System Interoperability Test Distance Protection Response Time

\begin{tabular}{|c|c|c|c|c|c|c|c|c|c|}
\hline & \multicolumn{3}{|c|}{ Average (ms) } & \multicolumn{3}{|c|}{$\begin{array}{l}\text { Standard Deviation } \\
(\mathrm{ms})\end{array}$} & \multicolumn{3}{|c|}{ Maximum (ms) } \\
\hline & Zone1 & Zone2 & Zone3 & Zone1 & Zone & Zone3 & Zone1 & Zone2 & Zone? \\
\hline \begin{tabular}{|c|} 
A- \\
IED + \\
A-MU
\end{tabular} & 48.22 & 568.82 & 1036.42 & 6.989 & 5.298 & 4.143 & 56.208 & 576.115 & 1040 \\
\hline \begin{tabular}{|c|}
$\mathrm{A}-$ \\
$\mathrm{IED}+$ \\
$\mathrm{B}-\mathrm{MU}$
\end{tabular} & 52.46 & 572.14 & 1042.9 & 2.027 & 25.17 & 3.534 & 54.872 & 8.313 & 1046. \\
\hline \begin{tabular}{|c|}
$\mathrm{A}-$ \\
$\mathrm{IED}+$ \\
$\mathrm{C}-\mathrm{MU}$ \\
\end{tabular} & 49.3 & 562.28 & 1030.5 & 4.492 & 6.66 & 4.216 & 54.781 & 570.944 & 1035. \\
\hline $\begin{array}{c}\text { C-IED } \\
+ \\
\text { A-MU }\end{array}$ & 33.96 & 531.98 & 1028.58 & 5.069 & 6.761 & 4.484 & 40.024 & 539.764 & 1034.0 \\
\hline $\begin{array}{c}\text { C-IED } \\
+ \\
\text { B-MU }\end{array}$ & 37.9 & 542.4 & 1030.27 & 17.734 & 6.881 & 3.756 & 56.642 & 550.278 & 1035.6 \\
\hline $\begin{array}{c}\mathrm{C}-\mathrm{IED} \\
+ \\
\mathrm{C}-\mathrm{MU}\end{array}$ & 31.18 & 532.82 & 1027.32 & 4.575 & 2.362 & 2.696 & 35.788 & 534.182 & 1030.2 \\
\hline $\begin{array}{l}\text { Conv- } \\
\text { ention } \\
\text { al }\end{array}$ & 47.317 & 76.362 & 54.379 & 557.45 & 7.713 & 574.164 & 1019.933 & 3.558 & 1037.65 \\
\hline
\end{tabular}

During the test, A-MU and B-MU must be synchronised and the SmpSync value inside the packet must be is 1(local synchronised) or 2 (global synchronised) so the A-IED can get the SV data. Note: from a physical perspective, the SFP modules for fibre cable connections in the IEDs from different vendors are not compatible with each other.

The results show, as compared to the conventional relay performance, A-IED takes longer to perform the protection function with MUs from different manufacturers. This is because the MU takes time to process the analogue signals to generate SVs. C-IED has a faster performance than both AIED and conventional relay. In all the scenarios studied the GOOSE trip message was correctly received within the appropriate protection time.
Comparing the conventional and process bus approach, for Zone 1 and Zone 2, the average trip time of conventional relay is faster than A-IED with three MUs, but slower than C-IED. For Zone 3, the average trip time of conventional relay is quicker than the process bus approach. The maximum trip time for the three zones is longer than the maximum trip time of the process bus approach.

\section{Network Architecture Test with Parallel Redundancy Protocol (PRP)}

When no background traffic is within the network, the average travel time of SV packet is $81.234 \mu \mathrm{s}$. The result is based on a $10 \mathrm{~s}$ capture of the network traffic.

\section{1): Unicast Traffic}

The travel delay when injecting unicast traffic at $\mathrm{LAN}_{2}$ is recorded in TABLE $\mathrm{V}$ below.

$$
\text { TABLE V }
$$

SV packet delay in PRP network $(\mu \mathrm{s})$

\begin{tabular}{|c|c|c|c|c|c|c|c|c|}
\hline & \multicolumn{4}{|c|}{ Average $(\mu \mathrm{s})$} & \multicolumn{4}{|c|}{ Maximum $(\mu \mathrm{s})$} \\
\hline $\begin{array}{c}\text { Background } \\
\text { Traffic }(\mathrm{Mb} / \mathrm{s})\end{array}$ & 80 & 100 & 120 & 200 & 80 & 100 & 120 & 200 \\
\hline $\begin{array}{c}\text { With LAN } \\
1\end{array}$ & 94.917 & 94.924 & 94.925 & 94.919 & 141.223 & 156.946 & 160.716 & 163.37 \\
\hline $\begin{array}{c}\text { Without } \\
\text { LAN }_{1}\end{array}$ & 117.231 & 2362.24 & 2363.30 & 2364.42 & 159.636 & 2643.87 & 2644.68 & 2645.8 \\
\hline
\end{tabular}

To check the architecture influence on the SV transmission, the result with $\mathrm{LAN}_{1}$ and without $\mathrm{LAN}_{1}$ was recorded for comparison. The results show when $\mathrm{LAN}_{1}$ is connected, the time for SV travelling is not influenced by the traffic amount in $\mathrm{LAN}_{2}$. The $\mathrm{SV}$ going through $\mathrm{LAN}_{1}$ is faster than the one in $\mathrm{LAN}_{2}$ and thus the SV from $\mathrm{LAN}_{2}$ are discarded at the Red Box 2 output port. When $\mathrm{LAN}_{1}$ is not connected, the travelling time increases as the background traffic grows. However, when the traffic increases to more than $100 \mathrm{Mb} / \mathrm{s}$, the travelling time does not increase significantly, and stays at $2.36 \mathrm{~ms}$. This is because the buffer of the switches is overloaded and the excess part is discarded directly rather than queuing for transmission. When the network is full, a certain number of SV packets get lost. The results are based on the SV packets that are not lost in the Ethernet switch buffer when the network is fully loaded.

\section{2): Multicast traffic}

The results in Table VI show that the multicast traffic has an effect on both LANs. When the traffic grows, the delay increases with the traffic. However, when the bandwidth is fully occupied, the time is about the same, which is about $3 \mathrm{~ms}$. The SV packets delay when mixed with multicast traffic is longer than those mixed with unicast traffic; this is because the multicast traffic floods all the switches including the Red Box while the unicast traffic only exists in the Ethernet switch $1 \& 2$.

TABLE VI

SV packet delay in PRP network $(\mu s)$

\begin{tabular}{|c|c|c|c|c|c|c|c|c|}
\hline & \multicolumn{4}{|c|}{ Average $(\mu \mathrm{s})$} & \multicolumn{4}{|c|}{ Maximum $(\mu \mathrm{s})$} \\
\hline $\begin{array}{c}\text { Background } \\
\text { Traffic }(\mathrm{Mb} / \mathrm{s})\end{array}$ & 80 & 100 & 120 & 200 & 80 & 100 & 120 & 200 \\
\hline $\mathrm{LAN}_{1}$ & 101.01 & 2978.7 & 2979.1 & 2979.3 & 175.44 & 3283.97 & 3283.46 & 3290.02 \\
\hline $\mathrm{LAN}_{2}$ & 100.13 & 2960.8 & 2959.9 & 2960.8 & 162.74 & 3315.41 & 3644.39 & 3600.57 \\
\hline
\end{tabular}

\section{E. Sampled Value Loss Impact on IEDs}

The SV loss rate setting is related to Process Bus network performance, in particular, the possibility of loss of samples. 
The IED calculates a frame loss rate every cycle for each MU with which it communicates. The set threshold is an upper limit for the loss of Sampled Values from any of the Logical Nodes configured in the IED, calculated every cycle. If the loss rate level exceeds the set value, the IED generates the '92 Sample Alarm' and the related protection functions are inhibited.

\section{1) : A-IED and A-MU}

The loss rate level has a default setting of $10 \%$ in the IED system. The IED has an indication called SAV Absence to indicate whether the IED has received the SV streams which the IED is subscribing to. As the loss rate of SV increases, the reading of the IED changes. As shown in TABLE VII, when the loss rate is within 5\% the IED can still trip correctly for a Zone1 fault, the average trip time is $54.68 \mathrm{~ms}$, longer than the trip time when SV does not lose any packets. The longer time is because the traffic impairment increases the SV transmission time from MU to IED. When the loss rate increases from $5 \%$ to $28 \%$, the IED can read voltage and current values but the IED is in idle mode and is blind to the command. If the loss is larger than $28 \%$, the relay cannot read the current and voltage values but the $S A V$ Absence parameter is 0 which means the relay receives SV streams. Once the loss rate grows over $42 \%$, the $S A V$ Absence value changes from 0 to 1 and IED loses the SV stream. The result shows the $S A V$ Absence parameter does not indicate the IED is working correctly, because when the SV loses packets, SAV Absence shows the IED receives SV but the IED cannot display the SV value.

TABLE VII

A-MU SV Loss Impact on A-IED

\begin{tabular}{|c|c|}
\hline Loss Rate & Impact on IEDs \\
\hline Less than 5\% & Still trip correctly but takes longer time \\
\hline $5 \%-28 \%$ & IED is idle \\
\hline $28 \%-42 \%$ & Receiving SV but no display \\
\hline Over $42 \%$ & SV stream is lost \\
\hline
\end{tabular}

\section{2) : A-IED and $B-M U$}

As shown in TABLE VIII, when A-IED is connected to BMU, the IED allows up to a $3 \%$ SV loss and up to this level is still able to work correctly. When the loss rate is $3 \%$, the GOOSE trip signal is not normal for a Zone 1 fault, the Zone 1 trip occasionally needs more than $80 \mathrm{~ms}$ to publish, and the data value of the trip signal is not continuously issued. When the fault is permanent, the trip signal only lasts for $40 \mathrm{~ms}$ and then goes off. Sometimes, the IED trips twice for a permanent fault. When the loss is larger than $3 \%$ but less than $18 \%$, the IED becomes idle. If the loss is larger than $18 \%$, the $S A V$ Absence is still 0 but no SV are read. The limit value where $S A V$ Absence maintains value 0 is $45 \%$; above this value $S A V$ Absence changes to 1 .

TABLE VIII

B-MU SV Loss Impact on A-IED

\begin{tabular}{|c|c|}
\hline Loss Rate & Impact on IEDs \\
\hline Less than 3\% & Normal working state \\
\hline $3 \%-18 \%$ & IED becomes idle \\
\hline $18 \%-45 \%$ & Receiving SV but no display \\
\hline Over 45\% & SV stream is lost \\
\hline
\end{tabular}

\section{3) : A-IED and C-MU}

As shown in TABLE IX, when A-IED is connected to C$\mathrm{MU}$, a $58 \%$ loss rate is the limit when the $S A V$ Absence value is held at 0 . To read the correct voltage and current value, the loss rate limit is $17 \%$. However, when the loss is less than $17 \%$ but greater than $8 \%$, the IED becomes idle; the IED can trip with SV whose loss rate is less than $8 \%$, but the voltage reading is $207 \mathrm{kV}$ when it should be $225 \mathrm{kV}$. When the loss rate is between $3 \%$ and $8 \%$, the voltage value is between $170 \mathrm{kV}$ to $200.3 \mathrm{kV}$; when the loss rate is less than $3 \%$, the voltage reading becomes correct again.

For the Zone 1 fault, the trip signal is similar to the situation where the B-MU is connected to the A-IED. The trip signal does not last as long as the fault and sometimes trips more than once for the same fault.

TABLE IX

C-MU SV Loss Impact on A-IED

\begin{tabular}{|c|c|}
\hline Loss Rate & Impact on IEDs \\
\hline Less than 3\% & Normal working state \\
\hline $3 \%-8 \%$ & Display Error \\
\hline $8 \%-17 \%$ & IED becomes idle \\
\hline $17 \%-58 \%$ & Receiving SV but no display \\
\hline Over 58\% & SV stream is lost \\
\hline
\end{tabular}

4) : C-IED and A-MU

The C-IED performs differently from A-IED. The IED displays the SV on the screen, irrespective of how many SV packets are lost. However, the reading is not stable as shown in TABLE $X$. The normal reading for the secondary side voltage is $63.5 \mathrm{~V}$. The average trip time for a Zone 1 fault is $84.2 \mathrm{~ms}$ with SV of $2 \%$ loss rate.

TABLE X

Impaired SV reading between C-IED and A-MU

\begin{tabular}{|c|c|}
\hline Loss Rate & IED reading (V) \\
\hline $80 \%$ & $9.11-19.91$ \\
\hline $40 \%$ & $15.27-43.42$ \\
\hline $20 \%$ & $44.44-56.5$ \\
\hline $5 \%$ & $56.31-62.47$ \\
\hline $2 \%$ & $61.09-62.14$ \\
\hline
\end{tabular}

5) : C-IED and $B-M U$

Unlike the A-MU, the C-MU has a variable reading when the loss is as small as $2 \%$, as shown in TABLE XI. No trip signal is issued from C-IED for a Zone1 fault with a $2 \%$ loss of packets.

TABLE XI

Impaired SV reading between C-IED and B-MU

\begin{tabular}{|c|c|}
\hline Loss Rate & IED reading (V) \\
\hline $80 \%$ & $3.49-10.59$ \\
\hline $40 \%$ & $18.95-33.47$ \\
\hline $20 \%$ & $36.72-40.32$ \\
\hline $5 \%$ & $45.42-53.41$ \\
\hline $2 \%$ & $53.47-63.04$ \\
\hline
\end{tabular}

6) : C-IED and C-MU

TABLE XII shows the impact of SV loss between C-MU and C-IED is similar to the situation where A-MU is connected to C-IED.

TABLE XII

Impaired SV reading between C-IED and C-MU

\begin{tabular}{|c|c|}
\hline Loss Rate & IED reading (V) \\
\hline $80 \%$ & $1.96-19.37$ \\
\hline $40 \%$ & $12.06-41.84$ \\
\hline $20 \%$ & $42.28-50.12$ \\
\hline $10 \%$ & $47.82-60.46$ \\
\hline $5 \%$ & $53.68-58.69$ \\
\hline $3 \%$ & $58.52-60.79$ \\
\hline $2 \%$ & $59.21-62.14$ \\
\hline
\end{tabular}




\begin{tabular}{|c|c|}
\hline $1 \%$ & 62.262 \\
\hline
\end{tabular}

From the results above we can see that C-IED does not tolerate a loss greater than $1 \%$. When the loss increases, the reading for SV on the IED varies with a wider range. When the loss rate is at $3 \%$, the vast majority of the trip signals occur around $34 \mathrm{~ms}$ for a Zone 1 fault but occasionally, the trip signal takes $566 \mathrm{~ms}$ to be issued, which is unacceptable.

\section{F. Frequency Response Capability of MUs}

From the results plotted in Fig. 14, A-MU and B-MU have a similar performance for different frequencies. When the frequency of the input signal is below $20 \mathrm{~Hz}$, the $\mathrm{SV}$ waveform from A-MU and B-MU are distorted. For $30 \mathrm{~Hz}$ and $40 \mathrm{~Hz}$, the voltage RMS value of SV streams is less than the input analogue voltage. The voltage reading becomes correct when the frequency is $50 \mathrm{~Hz}$ but as the frequency reaches $1000 \mathrm{~Hz}$, the ratio between the B-MU output voltage and the input voltage decreases from 1 to 0.9 , while 0.85 for A-MU. When the frequency goes above $2 \mathrm{kHz}$, the reading for both MUs is unstable. If the frequency is larger than $1000 \mathrm{~Hz}$, the SV waveform is in triangle waveform and the voltage RMS value becomes unstable when the frequency is above $2000 \mathrm{~Hz}$. Different from A-MU and B-MU, the voltage ratio for C-MU dramatically decreased from 1 at $50 \mathrm{~Hz}$ to nearly zero at $200 \mathrm{~Hz}$. When C-MU receives analogue input value at an operating frequency greater than $300 \mathrm{~Hz}$, the reading for output $\mathrm{SV}$ stream becomes unstable. C-MU cannot recover an analogue input of high frequencies.

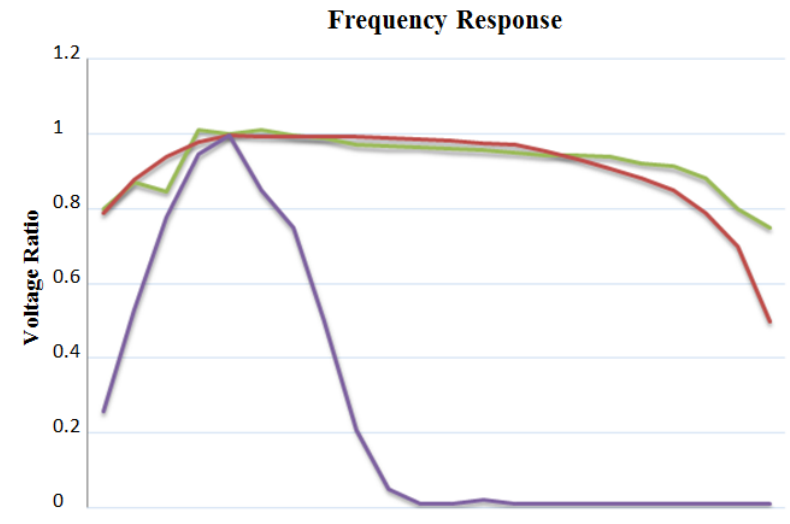

$1020304050607080901002003004005006007008009001000150020003000(\mathrm{~Hz})$

Fig. 14. Frequency response comparison between multivendor MUs.

\section{G. The Recovery of the Step Change Signal}

Fig. 15 shows the recovery of $100 \mathrm{~V}$ step-up signal by different MUs. The three MUs perform differently when recovering the same step change signal. The A-MU recovers the signal with correct magnitude. The step change transient is represented by $4 \mathrm{SV}$ packets. B-MU waveform correctly presents the step change transient; however, the amplitude drops down gradually to $0 \mathrm{~V}$. This is because the MU uses an interface to measure and digitise analogue voltage/current 75 value. The digitised signals are transmitted to MU for package and publication. The C-MU and interface of B-MU contain capacitor component so it cannot hold Vdc. Both B-MU and $\mathrm{C}-\mathrm{MU}$ are designed for Vac. C-MU takes $3 \mathrm{SV}$ packets to represent the step change transient and maintain the value for the next $4 \mathrm{SV}$ packets and then the signal dramatically drop to $0 \mathrm{~V}$. The interesting part is that the waveform has another pulse shape, as shown in Fig. 15 (c) with lower amplitude before it finally drops to $0 \mathrm{~V}$.

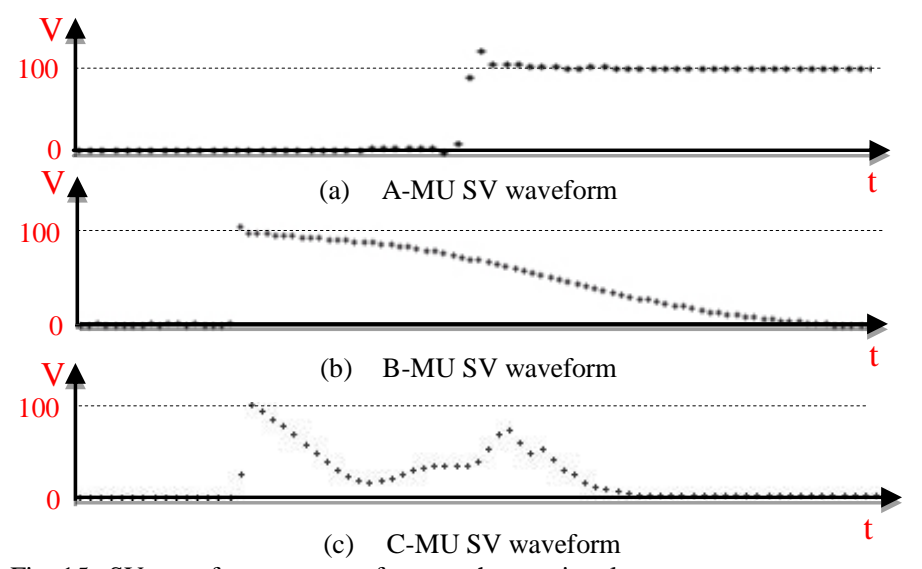

Fig. 15. SV waveform recovery for step change signal.

Apart from $100 \mathrm{~V}$, more voltage step are applied, the results are shown in Fig. 16 to Fig. 18.

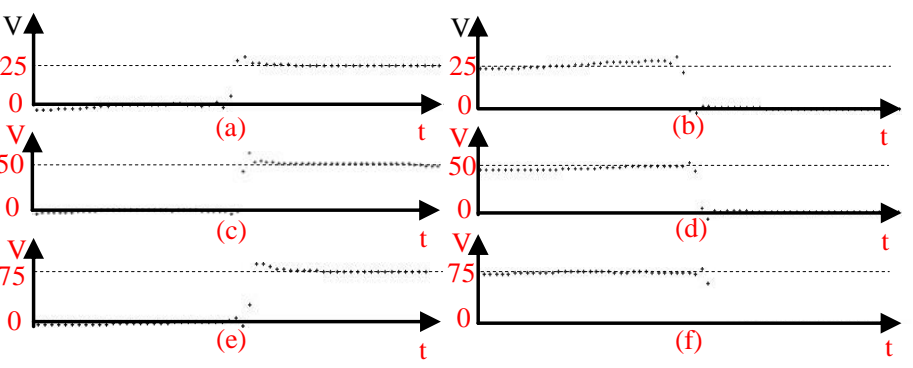

Fig. 16. A-MU step change signal recovery (a) $25 \mathrm{~V}$ step-up (b) $25 \mathrm{v}$ stepdown (c) $50 \mathrm{~V}$ step-up (d) $50 \mathrm{~V}$ step-down (e) $75 \mathrm{~V}$ step-up (f) $75 \mathrm{~V}$ step-down

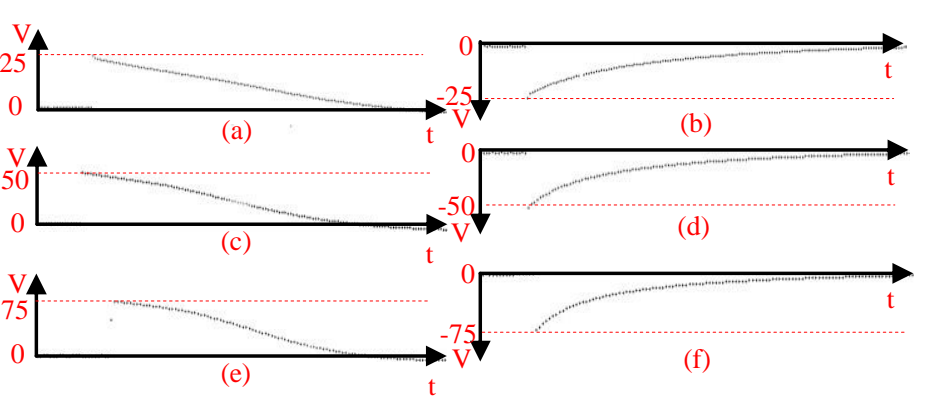

Fig. 17. B-MU step change signal recovery (a) $25 \mathrm{~V}$ step-up (b) $25 \mathrm{v}$ stepdown (c) $50 \mathrm{~V}$ step-up (d) $50 \mathrm{~V}$ step-down (e) $75 \mathrm{~V}$ step-up (f) $75 \mathrm{~V}$ step-down

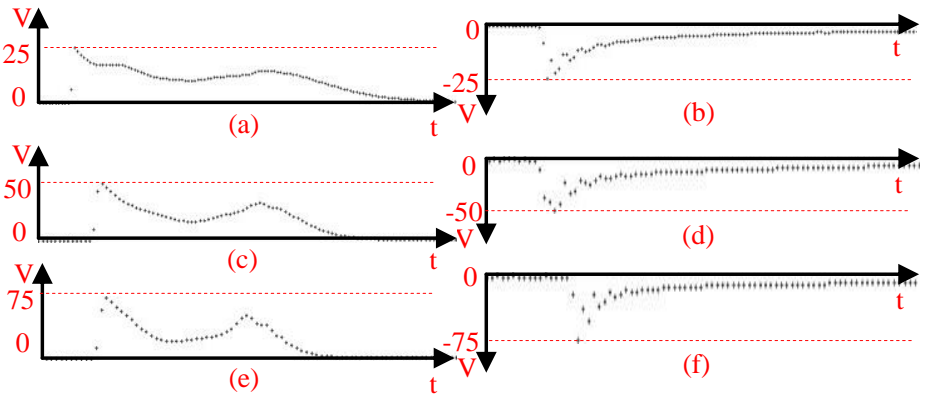

Fig. 18. C-MU step change signal recovery (a) $25 \mathrm{~V}$ step-up (b) $25 \mathrm{v}$ stepdown (c) $50 \mathrm{~V}$ step-up (d) $50 \mathrm{~V}$ step-down (e) $75 \mathrm{~V}$ step-up (f) $75 \mathrm{~V}$ step-down 
The step change recovery for $25 \mathrm{~V}, 50 \mathrm{~V}$ and $75 \mathrm{~V}$ are similar to $100 \mathrm{~V}$ for the three MUs respectively. B-MU has the quickest response. No packet drops between $0 \mathrm{~V}$ and the step voltage. For A-MU and C-MU, there are some packets representing the value in the transition area. Only A-MU can recover the Vdc value after the step change. B-MU and C-MU do not work properly when input is Vdc. When recovering the step down voltage, the output of A-MU and B-MU is symmetrical to that of step up voltage. For C-MU, the step down voltage recover is different from the step up voltage recovery. There are more packets that contain the value between $0 \mathrm{~V}$ and step voltage.

\section{H. Network Background Traffic Impact on IEDs}

As discussed in section E, the IED become idle when $5 \%$ of the SV packages are lost. The amount of background traffic is controlled by the inter frame time. When the background traffic inter-frame gap time of the transmission is less than $1600 \mathrm{~ns}$, the traffic takes up $94.77 \%$ of the bandwidth $100 \mathrm{Mb} / \mathrm{s}$. The next available setting in the traffic generator is $1520 \mathrm{~ns}$ inter frame time and the corresponding traffic amount is 95.39\%; for this setting some of the SV frames are lost. The reading on the A-IED varies from $100 \mathrm{~A}$ to $2 \mathrm{kA}$, for a normal $2 \mathrm{kA}$ primary current reading. The A-IED mal-operates when a Zone 1 fault is applied and sends several trip signals randomly. The more packets lost, the more random the trip signal is. The SV packets are captured by Wireshark and the lost rate is shown in TABLE XIII below.

\section{TABLE XIII}

SV loss rate when mixed with network traffic

\begin{tabular}{|c|c|c|}
\hline Inter frame Time( ns) & $\begin{array}{c}\text { Transmission Rate ( SV not } \\
\text { included) }\end{array}$ & SV loss rate \\
\hline 1600 & $94.77 \%$ & 0 \\
\hline 1520 & $95.39 \%$ & $3.7 \%$ \\
\hline 1440 & $96.32 \%$ & $3.85 \%$ \\
\hline 1280 & $97.30 \%$ & $5.86 \%$ \\
\hline 1200 & $97.97 \%$ & $6.9 \%$ \\
\hline 1120 & $98.64 \%$ & $8.45 \%$ \\
\hline 1040 & $99.32 \mathrm{v}$ & $9.15 \%$ \\
\hline 960 & $100 \%$ & $10.69 \%$ \\
\hline
\end{tabular}

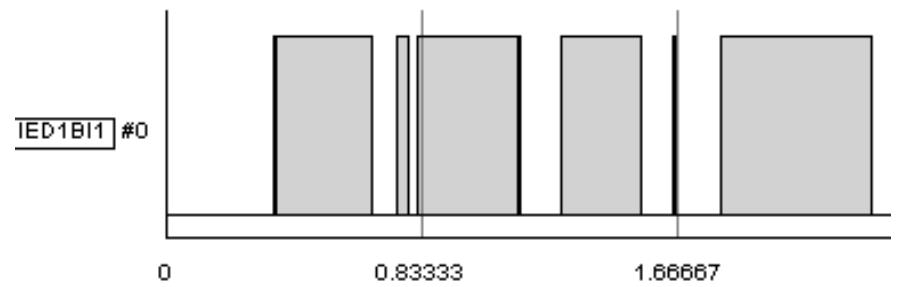

Fig. 19. GOOSE trip signal when SV lost rate is 3.7\%.

Fig. 19 is the RTDS record for GOOSE trip signals when the SV loss rate is at 3.7\%. A-IED trips multiple times for a permanent Zone 1 fault.

\section{Overwrite Impact on IEDs}

When the percentage of overwritten SV packets changes from $1 \%$ to $96 \%$, A-IED is able to display the correct voltage and current value. If more than $96 \%$ is modified, the current and voltage reading both become 0 and the $S A V$ Absence parameter value is turned to 1 . During this process, A-IED is not idle. The performance is different from the SV loss impact which makes IED become idle. The protection performance is not influenced and the trip time for faults in the three zones is the same as the correct SV. For C-IED, the address change of SV impact on C-IED is like the SV packet loss impact. When more than $5 \%$ of the SV multicast destination address is overwritten, the SV reading on the IED will vary, and the result is the same as section $\mathrm{E}$ when the $\mathrm{SV}$ is lost.

\section{J. Time Synchronisation Loss and Regain Impact on MUs}

When the time signal of the sampled value is lost, the sample count is not influenced. A-MU SmpSync change from global to local takes $14 \mathrm{~s}$ and from local to none takes 203s. BMU time sync status changes from global to none after 26s. CMU changes from local to none after 11 mins. When the time signal is regained, the time sync status of A-MU takes $18 \mathrm{~s}$ from none to global; B-MU takes 11s to change back from local to global while C-MU takes 36s to recover. One interesting observation when testing C-MU and A-IED is the impact of losing the time reference; the protection functions in A-IED continues to operate correctly for 11 minutes, including being able to clear an in-zone fault. Different from that, when $\mathrm{C}-\mathrm{MU}$ is connected with C-IED and the time reference signal is removed from the MU, the IED issued a GOOSE alarm, but the IED can still read the current and voltage signals forever, even if the MU activates a GPS alarm.

\section{CONCLUSION}

It is common to use IEDs and MUs from multi-vendors when replacing life-expired devices, especially when the original manufacture's device is not available. Interoperable components reduce time and costs associated with the integration into a complex system. It is necessary that tests are carried out before real implementation of a multi-vendor system in the substation. Real-time networks and precision timing were used to provide system level tests. The conventional relay performance was provided as a benchmark for the performance of the multi-vendor system. The MU performance during the processing and publishing period were measured with different time synchronisation signals. One of the MUs has longer processing and publishing times than the other two MUs. When dealing with signals of different frequencies, one of the MUs cannot accept low frequencies while the other two can respond to frequencies up to $1000 \mathrm{~Hz}$. MUs from different vendors were tested with various IEDs and the results show one of the IED trips longer than conventional relay. Testing of the SV network delay was carried out under both normal and extreme conditions to determine the limit of the operation. The two IEDs work well with different MUs in normal situation but when the SV packets are lost, their response are different from each other. The results also indicate the IEDs use different schemes to read the SV data as one of the IED has no reading whilst the other has an unstable reading for the same overwritten SV stream. The sensitivity of time synchronisation signal for MUs differ from each other during the losing and regaining period.

To perform the tests described in this paper, a large amount of time was spent configuring different MUs and IEDs since their configuration interface settings options vary. This further 
emphasizes the need for the configuration to be standardised by the utility. Further operating scenarios and more comprehensive test architectures, with additional vendor products will be investigated and the results presented here will provide confidence to organizations that consider adopting the technology that can meet their requirements. More comprehensive interoperability evaluation process needs to be conducted to provide assurance to the utility with the plug and play swapping of devices in an IEC61850 secondary system to provide a reliable and cost effective solution.

\section{APPENDIX}

Fig. 20 is the Single Line diagram for Zone 1 operation.

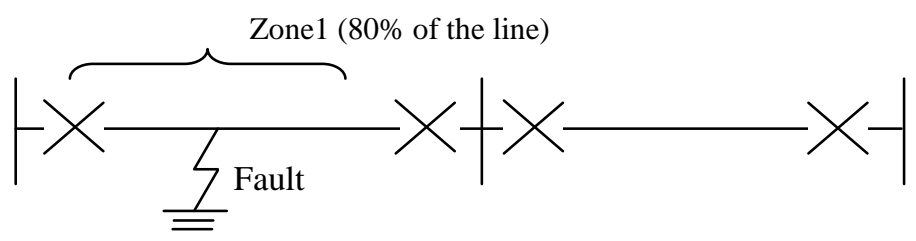

Fig. 20. Single line diagram for Zone1 operation.

\section{REFERENCES}

[1] A. Wen, J. Fitch, R. Zhang, P. Holliday, D. Barron, "A strategy for replacing electricity transmission protection and control systems to minimise the requirement for network outages," Asset Management Conference 2012, IET \& IAM , vol., no., pp.1,5, 27-28 Nov. 2012

[2] International Electrotechnical Commission. IEC 61850-9-2: 2011. "Communication networks and systems for power utility automation Part 9-2: Specific communication service mapping (SCSM) - Sampled values over ISO/IEC 8802-3". 2011.

[3] UCA International Users Group. IEC 61850 9-2 Light Edition. "Implementation Guideline for digital interface to instrument transformers using IEC 61850-9-2 R2-1". 2004.

[4] International Electrotechnical Commission. IEC 61850-8-1: 2011. "Communication networks and systems for power utility automation Part 8-1: Specific Communication Service Mapping (SCSM) Mappings to MMS (ISO 9506-1 and ISO 9506-2) and to ISO/IEC 88023". 2011.

[5] A. Apostolov, "Impact of IEC 61850 on the interoperability and reliability of protection schemes," 2013 IEEE Power and Energy Society General Meeting (PES), vol., no., pp.1,5, 21-25 July 2013

[6] H. Dawidczak, H. Englert, "IEC 61850 interoperability and use of flexible object modelling and naming"

[7] J.C. Tan, C. Zhang, Z.Q. Bo, "The importance of IEC 61850 interoperability testing" Universities Power Engineering Conference, 2008. UPEC 2008. 43rd International , vol., no., pp.1,5, 1-4 Sept. 2008

[8] M.G. Kanabar, T.S. Sidhu, "Performance of IEC 61850-9-2 Process Bus and Corrective Measure for Digital Relaying" IEEE Transactions on Power Delivery, , vol.26, no.2, pp.725,735, April 2011

[9] D.M.E. Ingram,.; P. Schaub, R.R. Taylor, D.A. Campbell, "SystemLevel Tests of Transformer Differential Protection Using an IEC 61850 Process Bus," IEEE Transactions on Power Delivery, vol.29, no.3, pp.1382,1389, June 2014

[10] L. Yang; P.A. Crossley, J. Zhao; H. Li, A. Wen, "Impact evaluation of IEC 61850 process bus architecture on numerical protection systems," Sustainable Power Generation and Supply, 2009. SUPERGEN '09. International Conference on, vol., no., pp.1,6, 6-7 April 2009

[11] L. Yang, P.A. Crossley, A. Wen, R. Chatfield, J. Wright, "Performance assessment of a IEC 61850-9-2 based protection scheme for a transmission substation," Innovative Smart Grid Technologies (ISGT Europe), 2011 2nd IEEE PES International Conference and Exhibition on , vol., no., pp.1,5, 5-7 Dec. 2011

[12] X. Sun, M. Redfern, P. Crossley, L. Yang; H. Li; U.B. Anombem, A. Wen; R. Chatfield, "IEC 61850:9-2 process bus architecture for substation protection schemes, "2011 International Conference on Advanced Power System Automation and Protection (APAP), , vol.2, no., pp.1373,1378, 16-20 Oct. 2011
[13] M. Mekkanen, R. Virrankoski, M.Elmusrati, E. Antila, "Analysis and methodology for measuring the IEC61850 GOOSE messages latency: Gaining interoperability testing" 2014 World Congress on Computer Applications and Information Systems (WCCAIS), vol., no., pp.1,6, 1719 Jan. 2014

[14] Y. Li. P.A. Crossley, A. Wen, R. Chatfield, J. Wright, "Design and Performance Testing of a Multivendor IEC61850-9-2 Process Bus Based Protection Scheme" IEEE Transactions on Smart Grid, vol.5, no.3, pp.1159,1164, May 2014

[15] K. Koshiishi, K. Kaneda, Y. Watabe, "Interoperability experience with IEC 61850-based Substation Automation Systems," 2012 IEEE PES Transmission and Distribution Conference and Exposition (T\&D), , vol., no., pp.1,5, 7-10 May 2012

[16] Institute of Electrical and Electronic Engineer. IEEE std 1588TM 2008. "IEEE Standard for Precision Clock Synchronization Protocol for Networked Measurement and Control Systems". New York: IEEE; 2008.

[17] International Electrotechnical Commission. IEC 62439-3: 2012. "Industrial communication networks - High availability automation networks - Part 3: Parallel Redundancy Protocol (PRP) and Highavailability Seamless Redundancy (HSR)". 2012.

[18] Ponovo MsgAnalysisSys V2.13, Software available from http://www.relaytest.com/.

[19] DAG 7.5G4 Card User Guide, Endace Technology Ltd, May 2012

[20] D.M.E. Ingram, P. Schaub, R.R. Taylor, D.A. Campbell, "Performance Analysis of IEC 61850 Sampled Value Process Bus Networks,", IEEE Transactions on Industrial Informatics, vol.9, no.3, pp.1445,1454, Aug. 2013

[21] D.M.E. Ingram, F. Steinhauser, C. Marinescu, R.R. Taylor, P. Schaub, D.A. Campbell, "Direct Evaluation of IEC 61850-9-2 Process Bus Network Performance," IEEE Transactions on Smart Grid, vol.3, no.4, pp.1853,1854, Dec. 2012.

[22] J. Breuer, V. Vojtěch, R. Jaroslav, "Precise packet delay measurement in an Ethernet network." Measurement 54 (2014): 215-221.

[23] MX123001A Data Quality Analyzer Control Software Operational manual, ANRITSU CORPORATION, 25 ${ }^{\text {th }}$ Edition, October 2012

[24] Technical Manual of MiCOM P543, P544, P545, P546 Current Differential/Distance Protection Relays by Alstom.

[25] GTFPI product information sheet. Available from https://www.rtds.com/the-simulator/our-hardware/gtfpi-card/

\section{BIOGRAPHIES}

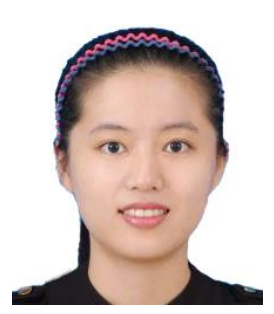

Xi Chen received BEng. Degree from the North China Electric Power University, and is studying at the University of Manchester as a PhD student. Her research interest included the IEC 61850 substation automation system, Interoperability between multi-vendor devices.

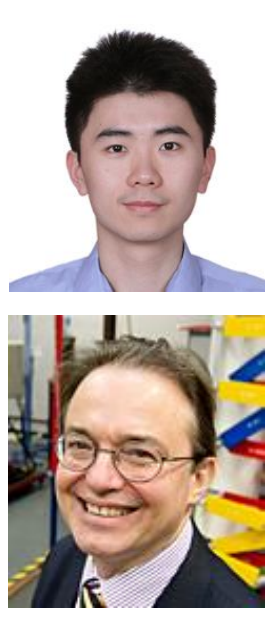

Hao Guo received the BEng. Degree from the University of Manchester, Manchester, U.K. in 2012. He is currently a $\mathrm{PhD}$ student at The University of Manchester. His research interests include time synchronization, Ethernet redundancy and engineering within power substations.

Peter Crossley received the B.Sc. degree from UMIST, Manchester, U.K., in 1977 and the Ph.D. degree from the University of Cambridge, U.K., in 1983. He is a Professor of Electrical Power Systems Engineering at The University of Manchester, U.K. and Director of Doctoral Training Centre. He has published over 250 technical papers on protection and is an active member of various CIGRE, IEEE, and IET committees. 